\title{
Towards a more culturally competent health care delivery system
}

\section{Commentary}

A consequence of living in ever-changing demographics, and a multicultural society is the necessity for cultural competency in health care delivery. In recent years, the emphasis on cultural competence has gained momentum involving innovative strategies to integrate skills, knowledge and attitudes, and behavior skills that include diverse methods for teaching and learning, required courses, distance learning, anthropological and cultural immersion experiences. To this end, it is critical to reiterate the dimensions of cultural competence to better equip health care providers, especially nurses at the forefront of care delivery, with the tools necessary to apply cultural competent skills and knowledge in their everyday lives, and the health care they deliver to culturally diverse populations.

Cultural competency is now a familiar phrase that continues to make headlines in the scientific literature. Many organizations, schools, and authors have emphasized its importance, effectiveness, and teaching theories. Variations, quite similar, of definitions for cultural competence abound the literature. Most of these highlight the need to be aware and responsive of patients' cultural views when providing health care to diverse populations. It is important to understand the terms 'culture' and 'competence' before attempting to explain cultural competence however. Mason' ${ }^{1}$ stated, "Culture was ever changing... [and] arriving at this end of the continuum was an ideal state requiring a lifelong commitment" (p. 6).

Culture also implies the "integrated patterns of human behavior that include thoughts, communications, actions, customs, beliefs, values, institutions of racial, ethnic, religious, or social groups" (OMH, 2005, para. 1). Mason, Cross, Rider and Friesen ${ }^{2}$ contended that cultural competence was "a developmental process for each worker, and ... not something that happens because one reads a book, attends a workshop or happens to be a member of a minority group" ( $p$. 4). Mason ${ }^{1}$ described five principles underlying cultural competence: valuing diversity, conducting a self-assessment, understanding the dynamics of difference, expanding cultural knowledge and resources, and adapting to diversity.

Mason ${ }^{1}$ believed that adapting to diversity involves the agencies or individuals making appropriate adjustments to attitudes, practices, structure, policies, and their environment. Awareness or the recognition of how one's own culture influenced how one thinks and acts is the second component of this cultural competence model. Cross, et al., ${ }^{3}$ posited that cultural competence was a "process" and "a set of congruent behaviors, attitudes and policies that come together in a system, agency or professional to work effectively in cross-cultural situations" (p. 297).

Cross et al., ${ }^{3}$ proposed one of the earliest models for cultural competence. The model focused on cultural competence at the organizational level, with elevated levels of understanding of the concept at the agency and provider levels. In addition, cultural competence is a developmental process, which moves along a continuum of six positions, ranging from least competent to most
Volume 5 Issue 6 - 2017

\author{
Judith-Jolie Mairs-Levy \\ The National Graduate School of Quality Management, Western \\ Governors University, USA
}

Correspondence: Judith-Jolie Mairs-Levy, The National Graduate School of Quality Management,Western Governors University, USA, Email phealthdoc@gmail.com

Received: April 30, 2017 | Published: May 10, 2017

competent. The least competent is cultural destructiveness, which includes attitudes and policies that are destructive to individuals, and reflect feelings of superiority, dominance, and control of one group over another. ${ }^{3}$

Cultural incapacity occupies the second position along the continuum, and while agencies and individuals in this position are not intentionally destructive; they lack the capacity to help culturally diverse clients or communities. As such, they tend to lean towards segregation, making minority groups feel unwelcomed. Cultural blindness is the third position, and is closer to the midpoint of the developmental process. On this continuum, although agencies and individuals in this position tend to think in an unbiased manner, they fail to acknowledge that culture makes a difference, and end up categorizing by treating everyone equally, instead of in an individualized manner acknowledging their culture. ${ }^{3}$

Along this continuum, and as individuals and agencies continue to develop, those in positions of cultural pre-competence realize their weaknesses in serving culturally diverse populations and will try to improve. In addition, they may even train their employees to become more culturally sensitive, hire more employees of visible minority groups, and conduct needs assessments on their clients so as to better serve them. ${ }^{3}$

\section{Conclusive thoughts}

It is paramount we do not become complacent or believe the accomplishment of any of the above-mentioned tasks is enough for becoming culturally competent. Instead, acquiring cultural competency takes time. It is an ongoing process, and one that requires continuous desire to expand one's knowledge base of cultural competent practice through research. This aspect is the most culturally proficient point along the continuum, requiring levels of solid understanding, acceptance, respect for and tolerance of differences, ongoing selfassessment, knowledge expansion, revisions, and modification of service to meet the needs of diverse patient populations.

Much is at stake for the future of health care in America: the implications for current and future education of health care providers, and the delivery of culturally competent health care to 
positively influence health outcomes are critical. There is much work ahead, however, if challenges are addressed appropriately, major developments in future innovations stand to experience a major shift. One that will move us closer towards public health's goal: of adequately addressing culturally competency through multicultural education and continued progress towards delivering culturally competent care to patients in keeping with America's changing demographics. ${ }^{4}$

\section{Acknowledgements}

None.

\section{Conflict of interest}

Author declares that there is no conflict of interest.

\section{References}

1. Mason J. Developing culturally competent organizations. Focal Point. 1994;8(2):1-8.

2. Mason J, Cross T, Rider M, et al. Services to Minority Populations: What does it mean to be a culturally competent professional? Focal Point. 1988;2(4):1-16.

3. Cross T, Bazron B, Dennis K, et al. Towards a culturally competent system of care. Georgetown University Child Development Center, Washington, USA; 1989.

4. Cultural Competence, U.S. Department of Health and Human Services, Office of Minority Health. 2005. 